\title{
A note on the instability and pattern formation of shrinkage cracks in viscoplastic soils
}

\author{
Manolis Veveakis ${ }^{\mathrm{a}, *}$, Thomas Poulet ${ }^{\mathrm{b}, \mathrm{c}}$ \\ ${ }^{a}$ Civil and Environmental Engineering, Duke University, Durham, NC 27708-0287, USA \\ ${ }^{b}$ CSIRO Mineral Resources, 26 Dick Perry Avenue, Kensington, WA 6151, Australia \\ ${ }^{c}$ School of Minerals and Energy Resources Engineering, UNSW, Kensington, NSW 2033, Australia
}

\begin{abstract}
In this note we present a theoretical study on the conditions for the onset of cracks, as well as the corresponding pattern formation, in saturated viscoplastic soils under isotropic loading (extension). The type of stress applied is left unspecified, to cover a variety of loadings including shrinkage due to dessication, isotropic thermal expansion, mechanical loading and so forth. By treating the saturated soil as rigid viscoplastic, we obtain a $2 \mathrm{D}$ extension of the Cnoidal Waves equations [1]. By numerically solving the corresponding boundary value problem, we retrieve conditions for the onset of cracking instability in 2D loading, and identify the characteristic spacing between cracks to be a length scale combining all the hydro-mechanical parameters of the problem. Finally, we show that in a rectangular slab of clay under isotropic extension, patterns of triangular, rectangular and hexagonal cracks can tessellate the domain, with the hexagonal pattern being the energetically favored, as it minimizes the free energy of the system.
\end{abstract}

Keywords: mud-cracks, hexagonal patterns, bifurcation analysis, cnoidal waves

\section{Introduction}

Mud-cracks are structures of cracks frequently met in clayey soils, as a result of shrinkage of the sediment due to multiphysically induced surface loads. Such surface loads could be developing for different reasons, including (a) dessication at the sediment-air interface (dessication cracks) 2], (b) generic expulsion of liquid from the structure (syneresis cracks) usually thermal shocks 5 . They usually form polygonal, interconnected networks of cracks and can be met in a variety of materials, other than soil, like paint films, biological tissues, ceramic glazes and so forth. Indeed, in a controlled, drying experiment performed on clayey silt formed into rectangular bars, Perron et al. 6] revealed two types of crack patterns depending on the geometry of the rectangular domain. When the domain was an essentially 1D bar (elongated rectangular slab), the soil was shown form parallel cracks to the short direction of the slab, as shown in Fig. 1. Alternatively, when the domain was square, polygonal patterns were forming (2), with the dominant polygons being a combination of squares (i.e. intersecting cracks at normal angles) and hexagons (i.e. intersecting cracks at 120 degree angles).

Apart from the puzzling structure these cracks form, mud-cracks are of profound importance in energy and environmental engineering [6. They strongly affect the soil's permeability, strength and compressibility, potentially compromising integrity of structures like embankments, dams, nuclear waste disposal sites and so forth [6]. Although the emergence of these cracks in brittle materials has been the subject of recent works [e.g 5 , little is known for the mechanisms controlling their formation in viscoplastic, clayey rich, saturated soils. In this work we present a short communication whereby such an approach is adopted, and conditions for the onset of cracking as well as their corresponding patterns are sought. Based on the recent theory of Cnoidal Waves in Solids [7, we study the soil as a biphasic mixture of solid and fluid, subject to tensile load caused by the shrinkage of the soil due to arbitrary (mechanical) surface loads.

\section{Mathematical modeling of isotropic extension of a saturated viscoplastic soil}

Motivated by the experiments performed by [6], shown in Figs. 1, 2, we start by idealizing the problem by considering a $2 \mathrm{D}$ slab of a fully saturated isotropic soil, subject to isotropic extension, as shown in Fig. 3 . The momentum and mass balance equations for such a configuration have been detailed in [7, 1] to be:

$$
\begin{array}{r}
\frac{\partial \sigma_{11}}{\partial x_{1}}=0, \frac{\partial \sigma_{22}}{\partial x_{2}}=0 \\
c_{v}\left(\frac{\partial^{2} p_{f}}{\partial x_{1}^{2}}+\frac{\partial^{2} p_{f}}{\partial x_{2}^{2}}\right)-\dot{\epsilon}_{v}=0
\end{array}
$$

${ }^{*}$ Corresponding Author

Email address: manolis.veveakis@duke.edu (Thomas Poulet) 


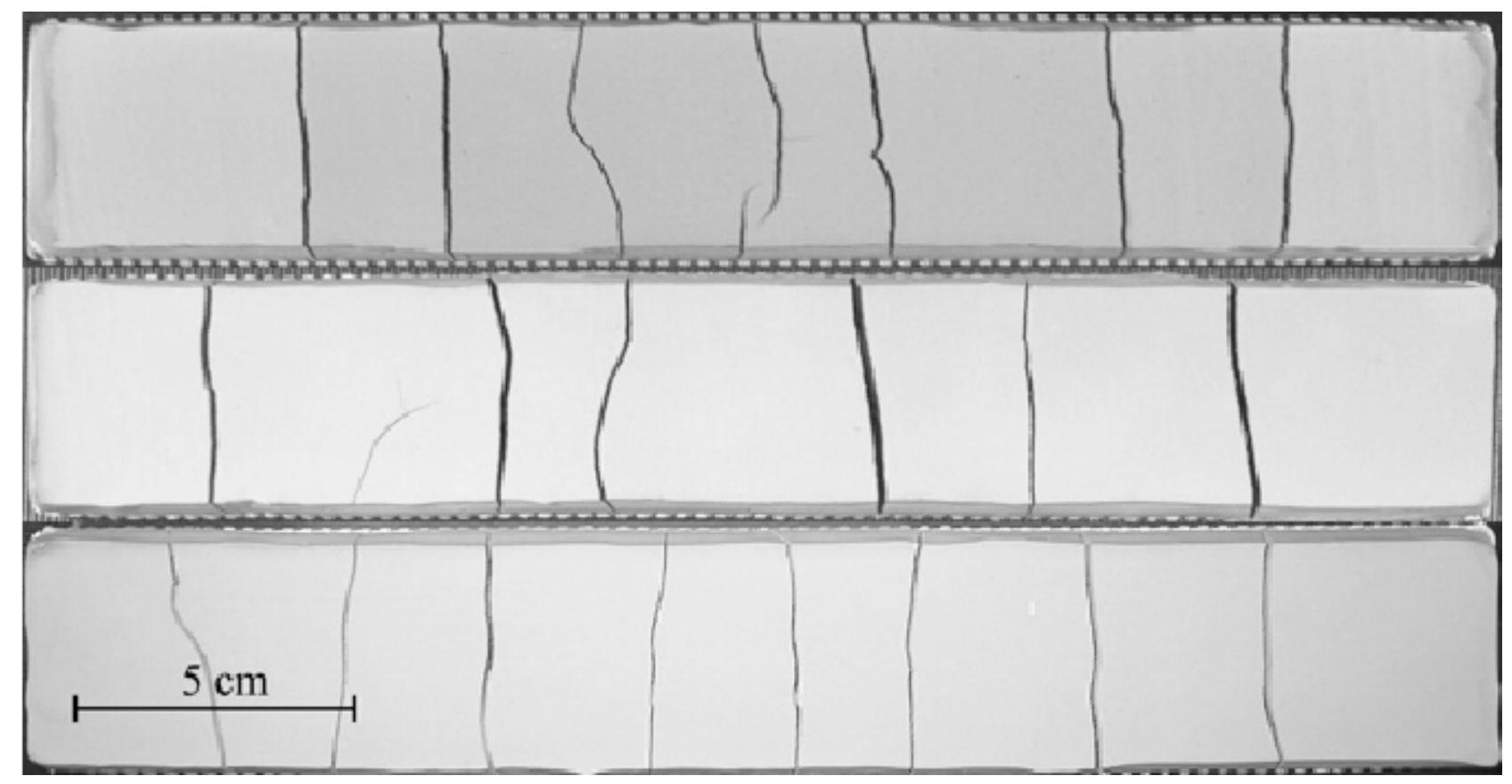

Figure 1: Drying experiment performed on constrained (through basal friction) clayey silt formed into rectangular bars, as reported by $[6$. Examples of desiccation crack one dimensional patterns in $20 \mathrm{~cm}$ long and $5 \mathrm{~cm}$ wide slabs. Figure reproduced from [6]
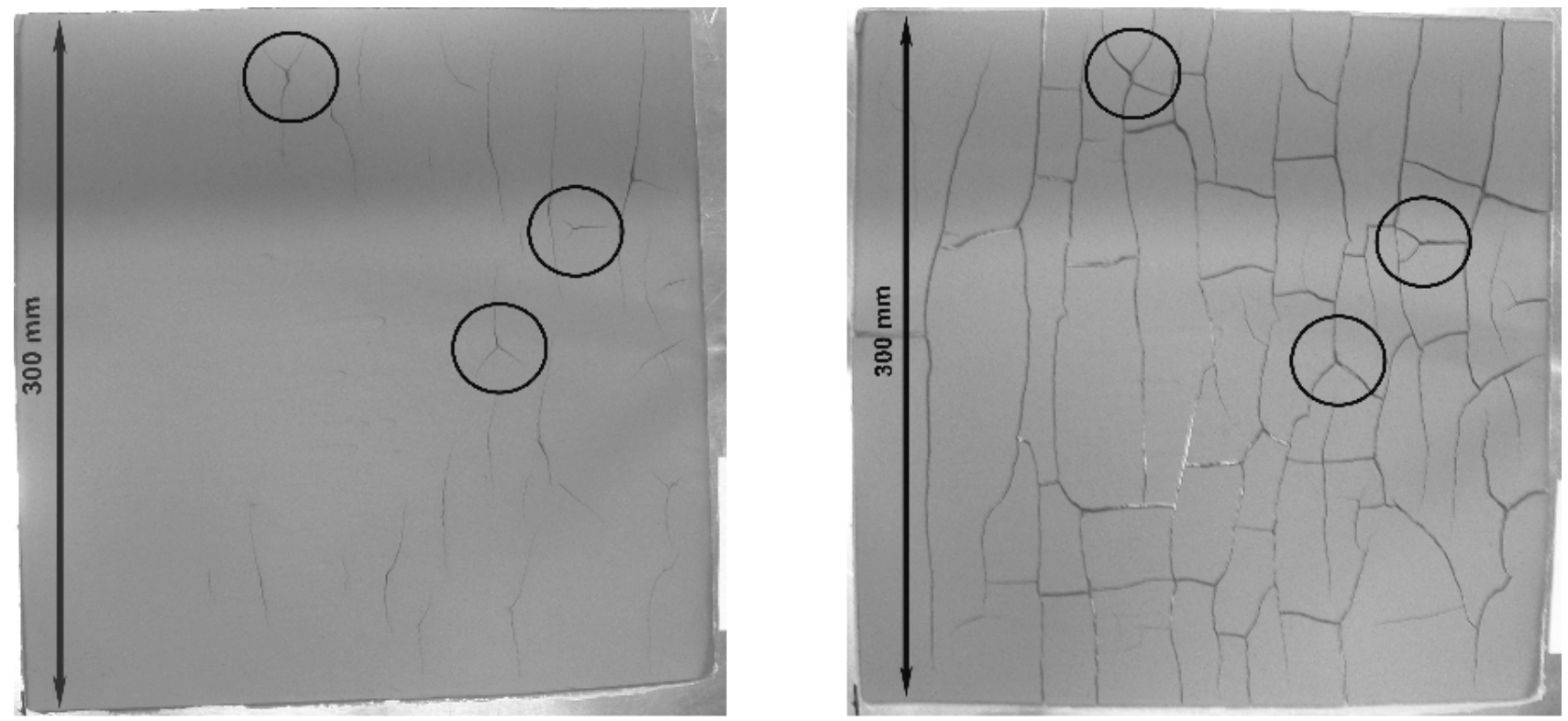

Figure 2: Drying experiment performed on constrained (through basal friction) clayey silt formed into 4 mm high rectangular bars, as reported by 6]. Examples of desiccation crack two dimensional patterns at (left) the initial stage of cracking: $120^{\circ}$ intercepting cracks circled; (right) the final stage of cracking: $120^{\circ}$ intercepting cracks circled. Notice that initially there are no normal angles formed, apart from the intersections with the boundaries, and the material is developing the tendency to form hexagons at $120^{\circ}$ angles. In contrast to that, the final configuration shows that normal angles prevail over the $120^{\circ}$ ones with both patterns being present simultaneously. Figure reproduced from [6] 


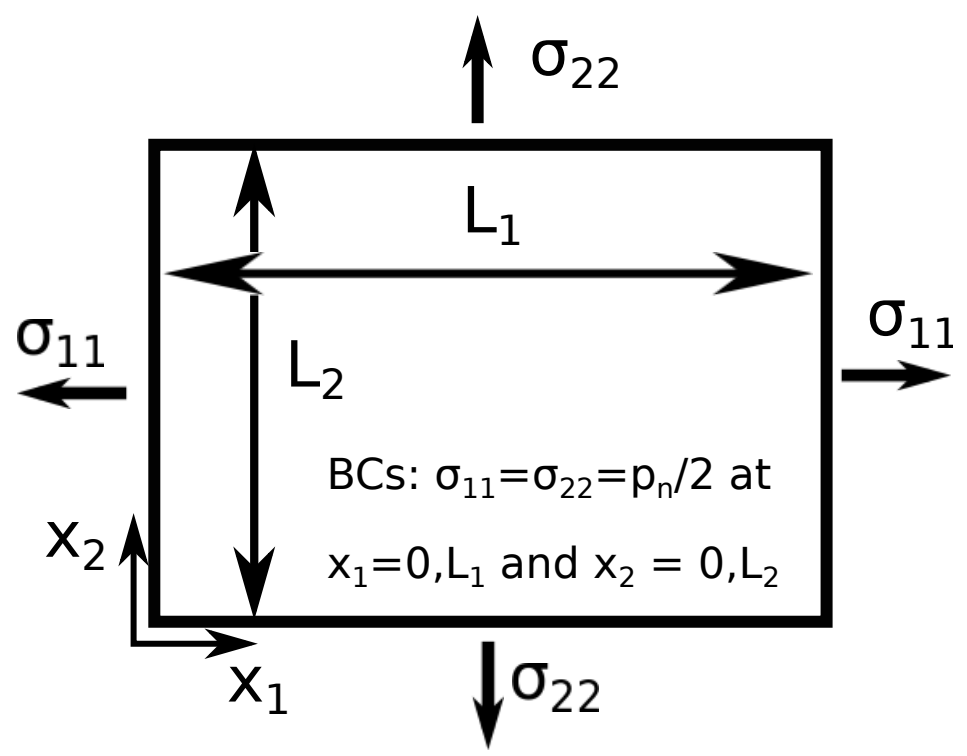

Figure 3: Problem description. A 2D slab of saturated viscoplastic soil is subject to isotropic extension.

where Darcy's law has been used for the fluid flux, with $p_{f}$ the pore fluid pressure and $c_{v}$ is a generalized consolidation coefficient (units $m^{2} s^{-1} \mathrm{~Pa}^{-1}$ ). In the case of isotropic extension, $\sigma_{11}=\sigma_{22}=p / 2$. By considering this, adding the first 2 equations and accepting: 1) Terzaghi's decomposition for the stress $\sigma_{i j}=\sigma_{i j}^{\prime}-p_{f} \delta_{i j}$ (prime denotes effective stress); and 2) the soil obeying a rigid viscoplastic rheology of the form: $\dot{\epsilon}_{v}^{p}=\dot{\epsilon}_{v}=\dot{\epsilon}_{n}\left(\frac{p^{\prime}-p_{Y}^{\prime}}{p_{n}^{\prime}-p_{Y}^{\prime}}\right)^{m}$; we may combine all equations in the final one, as obtained by [7, 1]:

$$
c_{v}\left(\frac{\partial^{2} p^{\prime}}{\partial x_{1}^{2}}+\frac{\partial^{2} p^{\prime}}{\partial x_{2}^{2}}\right)-\dot{\epsilon}_{n}\left(\frac{p^{\prime}-p_{Y}^{\prime}}{p_{n}^{\prime}-p_{Y}^{\prime}}\right)^{m}=0
$$

In the above expressions, $\dot{\epsilon}_{n}$ is the background (loading) strain rate, $p_{Y}^{\prime}$ is the (initial) yield value of the mean effective stress during isotropic extension, $p_{n}^{\prime}$ is the boundary (loading) value of the effective pressure and $m$ is the rate sensitivity exponent. Eq. (3) can be brought into the reduced, dimensionless form:

$$
\left(\frac{\partial^{2}}{\partial x^{2}}+\ell^{2} \frac{\partial^{2}}{\partial y^{2}}\right) \sigma-\lambda \sigma^{m}=0
$$

where the initial yield pressure is assumed constant, and

$$
\begin{array}{r}
x=\frac{x_{1}}{L_{1}}, y=\frac{x_{2}}{L_{2}}, \ell=\left(\frac{L_{1}}{L_{2}}\right), \sigma=\frac{p^{\prime}-p_{Y}^{\prime}}{p_{n}^{\prime}-p_{Y}^{\prime}} \\
\lambda=\frac{\dot{\epsilon}_{n} L_{1}^{2}}{c_{v}\left(p_{n}^{\prime}-p_{Y}^{\prime}\right)}
\end{array}
$$

The equivalent boundary conditions in dimensionless form are correspondingly, $\sigma=1$ when $x=0, x=1$ or $y=0, y=1$.

\section{Onset of cracks and pattern formation}

It appears that crack formation locations and circumstances, as well formation patterns can be interpreted with and hence, predicted by analyzing properties and singularities of the solution of the system of equations of equilibrium (Eq. 1) or its extensions and a combination of volume (mass) conservation law and a constitutive law (Eq. 4). In case of a domain with one side significantly larger than the other, like shown in Fig. 1. then Eq. (4) reduces to the 1D form of the cnoidal wave equation [7] along the long, $x$-direction (which is equivalent to setting $\ell \gg 1$ in Eq. 4, and taking first order expansion with $\sigma=$ const. in the $y$-direction):

$$
\frac{\partial^{2} \sigma}{\partial x^{2}}-\lambda \sigma^{m}=0
$$

subject to the boundary conditions $\sigma=1$ when $x=0, x=1$. This case has been extensively studied by [1, 7, 8, 9 analytically for $m=3$, and its solution is known as a cnoidal wave solution, because of the Jacobi $c n$-functions that constitute the base of the solution space. These $c n$-oidal functions, in brief are similar in spirit to trigonometric functions, except that their periodicity is based on elliptical, instead of circular basis. Cnoidal waves, compared to sine-waves are steeper near the peak and gentler near the bottom. Further to these studies, the results are generalized here for any value of $m$. To do so, Eq. (4) 

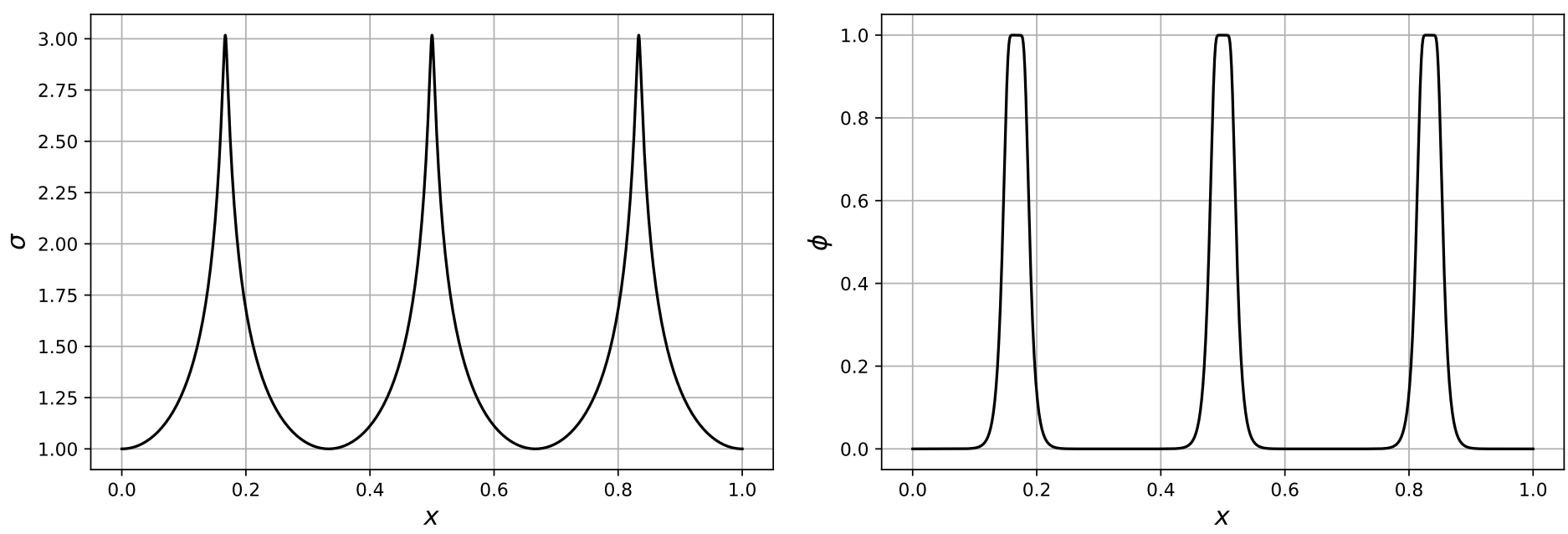

Figure 4: (left) Numerical solution of the 1D Eq. 6 for $m=5$ and $\lambda=46$, using the finite element code REDBACK 10, by enriching the standard finite element scheme with numerical regularization capping the stress singularities of the cnoidal equation [see 8 , for a physics based regularization approach]. We observe that three stress peaks are present, equidistantly placed inside the unit (normalized) domain. (right) Porosity profile, calculated as the solution of the storage equation $\dot{\phi}=(1-\phi) \dot{\epsilon}_{v}$. We notice that porosity increases in the positions of the stress peaks, pertaining to cracks forming equidistantly in the domain.

was implemented in the Finite Element simulator REDBACK[10, 11] and validated against the analytical solutions provided by [1. When $m=1$ the solution is the usual, diffusive front met for example at Terzaghi's $1 \mathrm{D}$ consolidation problem in compression. No instability is present and no cracks are therefore predicted to form when the soil is weakly rate dependent $(m=1)$. For any non-linearity in the flow law (exponents $m>1)$ the solution bifurcates for values of $\lambda$ larger than a critical value $\lambda_{c r}$. An example is given in [1, 7, 8, 9, for the case of $m=3$, where the solution presented stress singularities for $\lambda \geq \lambda_{c r} \approx 12.7$. From that value of $\lambda$ onward, the number of peaks increases with increasing $\lambda$ until obtaining a scaling where the number of peaks $N_{p} \sim \sqrt{\lambda}$ [1]. As we can see in Fig. 4 , in the 1D case the numerical solution indeed presents stress singularities that are periodically placed in the sample. These stress singularities correspond to porosity increase areas, i.e. cracks, that are appearing at the same locations with the stress singularities (Fig. 4 ).

These concepts can be generalized when the domain is square, i.e. when $\ell=1$ (or equivalently $L_{1}=L_{2}=L$ ) in Eq. (4). In this case Eq. (4) becomes:

$$
\frac{\partial^{2} \sigma}{\partial x^{2}}+\frac{\partial^{2} \sigma}{\partial y^{2}}-\lambda \sigma^{m}=0
$$

The main challenge in this case is that the problem at hand has no preferred directions. Indeed, no preferred geometrical directions can be identified in a square domain, unlike the 1D case above. Similarly, no preferred directions can be found from mechanical considerations due to the existence of the characteristics of the stress equilibrium equation, as one would typically do in a localization problem following the analysis of Hill [12. Since the problem at hand is isotropic extension of a mechanically isotropic soil, the characteristics of the stress equilibrium are imaginary [13] and therefore offer no preferred orientation for the crack patterns of Eq. (7) to be organized along. In this case, it is reasonable to argue that since there are no points or directions which are preferred in our domain, the entire domain must be tessellated into regular polygons (cells) with the domain's boundaries being surfaces of symmetry. Such complete and regular symmetry would require that the polygons be either equilateral triangles, squares or regular hexagons [see also 14. Since the triangular pattern is a sub-case of the hexagonal one, we will hereby study the two cases of squares and hexagons.

By stating that a certain periodic cell pattern is presented, we mean that there is a cell that repeats itself regularly; that the walls of the cell are surfaces of symmetry and that the cells occur contiguously. Mathematically, the only way of such a structure to tessellate exactly a square domain domain is to relax the Dirichlet boundary conditions and replace them with symmetric or mixed boundary conditions. Recalling that we are examining the case of isotropic extension as a loading state, we can consider an equivalent boundary value problem, whereby the square domain with side size $L$ is inscribed in a circle with diameter $L \sqrt{2}$ on which the isotropic loading is applied. Such a configuration would mean that the sides of the square boundary are surfaces of symmetry and strong, Dirichlet boundary conditions are only applied at the corners of the domain. Such a mathematical configuration would allow regular polygons to tessellate exactly the domain. The results of this approach are shown in Fig. 5, where the spacing $h / L$ between the sides of a square (Fig. 5 5 ) or two opposite sides of a hexagon (Fig. 5b) is shown to be the same as in the 1D case and is equal to the diameter of the circle inscribed in these cells.

Three questions are immediately emerging from the above considerations and call for further clarification: 1) What is the critical value of $\lambda, \lambda_{c r}$ for equidistant cracks to emerge as a function of the exponent $m$ ? 2) What is the normalized spacing $h / L$ depending on? and 3 ) which regular tessellation (triangles, squares, hexagons) would be preferable in nature?

Firstly, the critical value of $\lambda$ for cracks to emerge $\left(\lambda_{c r}\right)$ is a function of the exponent $m$, as shown in Fig 6 a. We notice 

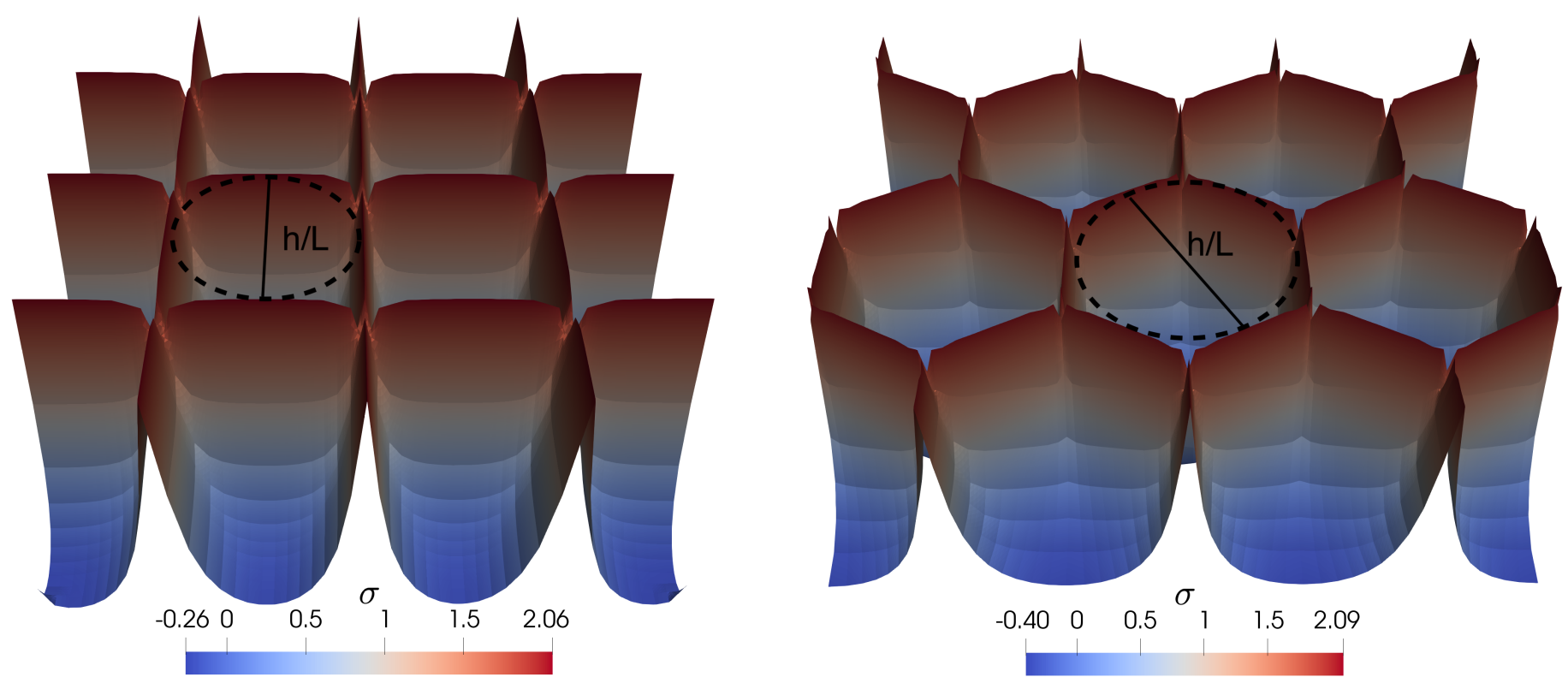

Figure 5: Numerical solution of the 2D Eq. 7 for $m=3$ and $\lambda=200$, using the finite element code REDBACK[10 with symmetry conditions at the sides of the square domain and Dirichlet boundary conditions at the corners. (left) Square patterns of stress peaks/cracks are forming, when the initial condition is perturbed using square pattern with a $0.1 \%$ stress perturbation. (right) Hexagonal patterns of stress peaks/cracks are forming, when the initial condition is perturbed using hexagonal pattern with a $0.1 \%$ stress perturbation. In both cases, the patterns are inscribing a circle of diameter $h / L$, obeying the spacing of Eq. 10

that $\lambda_{c r}$ is obeying an exponential law of the the form

$$
\lambda_{c r}=\lambda_{0} N_{p}^{2} e^{-0.2 m}, \text { for } m>1
$$

where $\lambda_{0}=12.7$ and $N_{p}$ is the number of peaks. By using the definition of the dimensionless group $\lambda$ (Eq. 5), this condition can be translated into a hydro-mechanical criterion for the onset of cracks in visco-plastic soils. Indeed, the solution bifurcates from the diffusive profile and cracks will be equidistantly placed when $\lambda>\lambda_{c r}$, i.e. when the following inequality is respected:

$$
\frac{\dot{\epsilon}_{n} L^{2}}{c_{v}\left(p_{n}^{\prime}-p_{Y}^{\prime}\right)}>\lambda_{0} N_{p}^{2} e^{-0.2 m}, \text { for } m>1
$$

The second point of interest is that these cracks are placed periodically in the domain, and their spacing is shown in Fig. $6 \mathrm{~b}$ to be following a root relationship with $\lambda$. As shown in Fig 6 , the normalized spacing (i.e. crack intensity) is:

$$
\frac{h}{L}=\frac{1}{N_{p}} \approx m^{-1 / 4} \sqrt{\frac{\lambda_{0}}{\lambda}}, \text { for } m>1
$$

where $\lambda_{0}=12.7$ is a constant.

To answer the third and final question (which pattern would be preferred in nature), we recall that processes in nature tend to minimize the free energy of the system. In the patterns examined above (triangles, squares and hexagons), the system would be required to distribute its free energy through these patterns. Since the pattern with the maximum surface area is the hexagonal one (with surface area of a unit cell $3 \sqrt{3} / 2$ versus 1 of a square and $\sqrt{3} / 4$ of an equilateral triangle), this pattern is the one that minimizes the system's free energy and would naturally be preferred. Note that similar considerations lead to hexagonal patterns being the preferred structure in a number of natural processes [see also 15, for a comprehensive review of natural processes self organizing in hexagonal structures]. However, because of the small difference in surface areas between all the patterns, as well as the fact that in nature the idealized geometrical and loading conditions assumed here are never met, it needs to be pointed out that all patterns, as well as combinations thereof are mathematically equiprobable as solutions of the system of equations.

\section{Conclusions}

We have shown in this work that by considering the isotropic extension of a slab of saturated viscoplastic soil, polygonal patterns of cracks emerge. The condition for the onset of instability, during which the solution bifurcates from the smooth diffusive profile, is given by Eq. (8), and the characteristic wavelength/spacing of the cracks is given by Eq (10). Both are functions of the generalized consolidation coefficient and yield pressure (or isotropic tensile strength) of the material, as well as the loading conditions (applied stress and strain rate) at hand. 

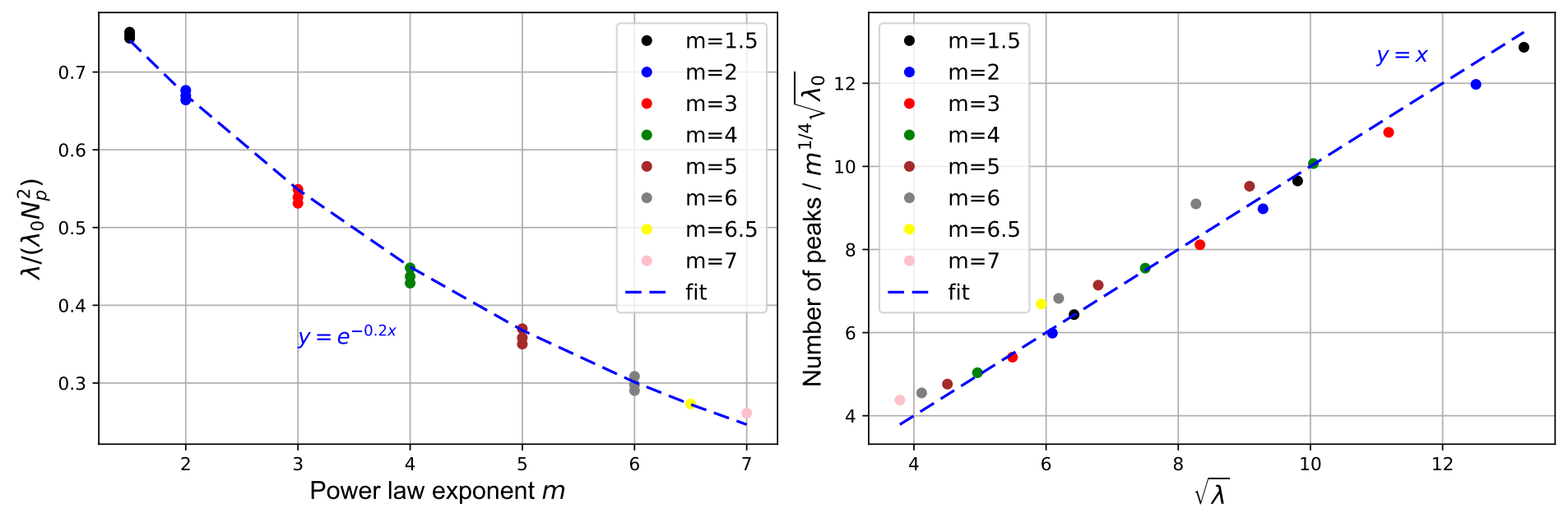

Figure 6: Global scaling of the two dimensional equation (7). (Left) Scaling of the critical value of $\lambda$ at which periodic stress peaks are spaced equidistantly (i.e. $h / L=1 / N_{p}$ ) in the domain, as a function of $m$. (Right) Scaling of the number of peaks (inverse intensity) as a function of $\lambda$, for a variety of values of $m$.

\section{Acknowledgements}

This research was partially supported by the DE-NE0008746-DoE project and the CSIRO-Duke University collaboration agreement.

\section{References}

[1] E. Veveakis, K. Regenauer-Lieb, Cnoidal waves in solids, Journal of the Mechanics and Physics of Solids 78 (0) (2015) 231 - 248. doi:http://dx.doi.org/10.1016/j.jmps.2015.02.010.

[2] E. M. Kindle, Some factors affecting the development of mud-cracks, The Journal of Geology 25 (2) (1917) 135-144.

[3] P. S. Plummer, V. A. Gostin, Shrinkage cracks; desiccation or synaeresis?, Journal of Sedimentary Research 51 (4) (1981) 1147-1156. doi:10.1306/212F7E4B-2B24-11D7-8648000102C1865D

[4] B. R. Pratt, Syneresis cracks: subaqueous shrinkage in argillaceous sediments caused by earthquake-induced dewatering, Sedimentary Geology 117 (1) (1998) 1 - 10. doi:https://doi.org/10.1016/S0037-0738(98)00023-2.

[5] B. Bourdin, J.-J. Marigo, C. Maurini, P. Sicsic, Morphogenesis and propagation of complex cracks induced by thermal shocks, Phys. Rev. Lett. 112 (2014) 014301. doi:10.1103/PhysRevLett.112.014301.

[6] H. Peron, L. Laloui, T. Hueckel, L. B. Hu, Desiccation cracking of soils, European Journal of Environmental and Civil Engineering 13 (7-8) (2009) 869-888. doi:10.1080/19648189.2009.9693159.

[7] E. Veveakis, K. Regenauer-Lieb, R. Weinberg, Ductile compaction of partially molten rocks: the effect of non-linear viscous rheology on instability and segregation, Geophysical Journal International 200 (1) (2015) 519-523. doi:10. $1093 / \mathrm{gji} / \mathrm{ggu} 412$

[8] S. Alevizos, T. Poulet, M. Sari, M. Lesueur, K. Regenauer-Lieb, M. Veveakis, A framework for fracture network formation in overpressurised impermeable shale: Deformability versus diagenesis, Rock Mechanics and Rock Engineering 50 (3) (2017) 689-703. doi:10.1007/s00603-016-0996-y.

[9] U. Kelka, M. Veveakis, D. Koehn, N. Beaudoin, Zebra rocks: compaction waves create ore deposits, Scientific Reports 7 (1) (2017) 14260.

[10] T. Poulet, M. Veveakis, A viscoplastic approach for pore collapse in saturated soft rocks using redback: An opensource parallel simulator for rock mechanics with dissipative feedbacks, Computers and Geotechnics 74 (2016) 211 - 221. doi:10.1016/j.compgeo.2015.12.015

[11] T. Poulet, M. Paesold, E. Veveakis, Multi-physics modelling of fault mechanics using redback - a parallel open-source simulator for tightly coupled problems, Rock Mechanics and Rock Engineering 50 (3) (2017) 733-749. doi:10.1007/ s00603-016-0927-y.

[12] R. Hill, The Mathematical Theory of Plasticity, Oxford University Press, London, 1950. 
[13] M. Paesold, A. Bassom, K. Regenauer-Lieb, M. Veveakis, Conditions for the localisation of plastic deformation in temperature sensitive viscoplastic materials, Journal of Mechanics of Materials and Structures 11 (2) (2016) 113-136.

[14] S. Chandrasekhar, Hydrodynamic and hydromagnetic stability, Dover Publications Inc., 1961.

${ }_{115}$ [15] P. Ball, Patterns in Nature: Why the Natural World Looks the Way It Does, University of CHicago Press, 1427 East 60th Street Chicago, IL 60637 U.S.A., 2016. 